\title{
Un Cadavre qui donne des " signes de vie »
}

Le cas de l'enfant mort-né au sanctuaire à répit

A corpse showing signs of life. The case of the stillborn child and the respite sanctuary

Jacques Gélis

\section{OpenEdition \\ Journals}

Édition électronique

URL : https://journals.openedition.org/tc/7073

DOI : $10.4000 /$ tc. 7073

ISBN : $1952-420 X$

ISSN : 1952-420X

Éditeur

Éditions de l'EHESS

\section{Édition imprimée}

Date de publication : 19 juin 2013

Pagination : 44-59

ISBN : 978-2-7351-1637-9

ISSN : 0248-6016

Référence électronique

Jacques Gélis, « Un Cadavre qui donne des « signes de vie » », Techniques \& Culture [En ligne], 60 |

2013, mis en ligne le 19 juin 2016, consulté le 29 septembre 2022. URL : http://

journals.openedition.org/tc/7073; DOI : https://doi.org/10.4000/tc.7073 


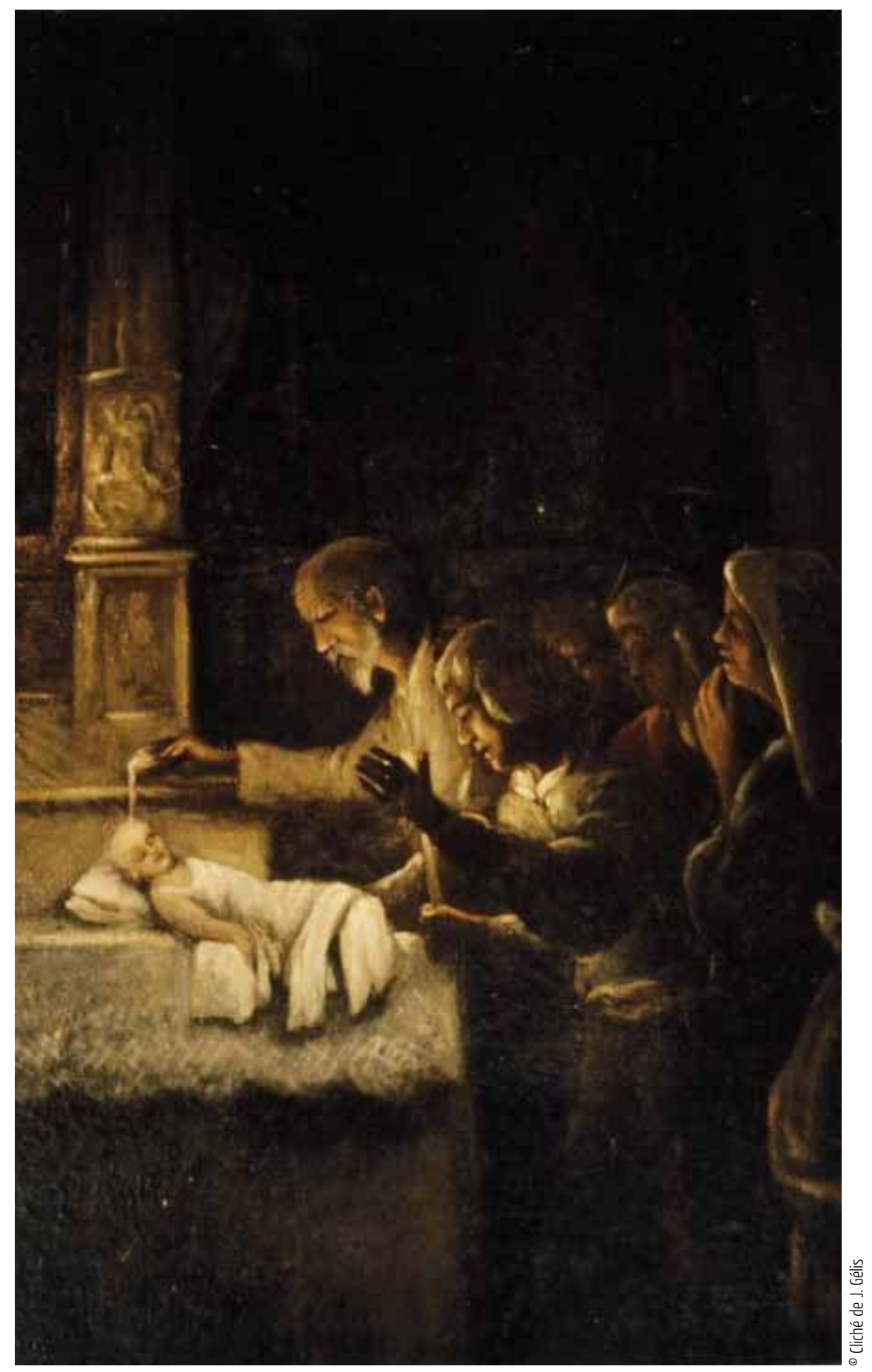




\section{UN CADAVRE QUI DONNE DES «SIGNES DE VIE»}

\section{Le cas de l'enfant mort-né au sanctuaire à répit}

Le nouveau-né est le symbole même de l'innocence et de la fragilité et l'on sait le drame que constitue aujourd'hui la naissance d'un enfant mort-né: le fruit mort avant même d'avoir vécu, la désespérance des parents, le sentiment d'avoir commis quelque erreur, la culpabilisation..

Or, l'arrivée d'un enfant mort, alors qu'on attendait de lui le prolongement du couple et de la lignée, était sans doute encore plus vivement ressentie aux siècles passés, au temps du catholicisme triomphant. Loin d'être insensibles à un tel drame, les parents redoutaient le sort qui attendait l'enfant mort sans baptême, puisque l'absence du sacrement qui sauvait à la vie éternelle vouait l'innocent au Limbe des enfants (Limbus puerorum), cet espace de souffrance où il était privé de la vision de Dieu: la peine du Dam. Son âme était destinée à errer pour l'éternité et à venir importuner les vivants. Quant à son corps, interdit de sépulture dans l'espace communautaire, il était enterré dans un jardin, un champ ou un pré, comme une bête... Telles étaient alors les « justices de l'au-delà » et l'on peut comprendre que les parents aient tout fait pour que l'enfant échappe à sa triste destinée. Le rituel aujourd'hui bien connu du « répit » résultait précisément de l'impossibilité pour les parents de faire le deuil de l'enfant. La décision ecclésiastique d'écarter toute possibilité de baptême du mort-né et d'assurer le déroulement ordinaire des rites d'ensevelissement suscitait une conduite de contournement. Il fallait que le disparu puisse être en repos et pour cela remplacer ce que l'on n'avait pu avoir par « quelque chose » d'autre.

Il restait en effet une issue, même si elle s'avérait bien aléatoire: exposer le cadavre de l'enfant devant une « image » miraculeuse. On attendait alors que des « signes de vie » 
sous trois pieds de terre quand alternant prières et protestations, elle finit par venir à bout des réticences de son époux. L'enfant est déterré, et c'est alors que l'on constate, à la stupéfaction générale, que le corps loin d'avoir été miné par la décomposition est en parfait état:

«Ils trouvèrent ce petit poupon beau, d'une couleur naturelle, vive et vermeille comme une rose. Il n'y avoit aucune marque de mort en tout son corps, ny aucune foulure ny blessure, sauf en une des mâchoires qui sembloit estre un peu froissée de terre. Jamais personne ne fut plus estonné que furent ces bonnes gens à ce spectacle; il ne savoient s'ils devoient rire ou pleurer, dans leur estonnement, ny croire ce qu'ils voioient ». (Maillard 1651)

Il faut bien entendu faire la part de l'hagiographie à la lecture d'un tel témoignage, mais nul doute que de nombreux enfants furent ainsi enterrés puis déterrés. Après une brève toilette à la petite dépouille, le père la met dans un sac ou dans un panier et, accompagné de l'accoucheuse, d'une ou deux voisines et d'une parente, il s'empresse de prendre le chemin du sanctuaire.

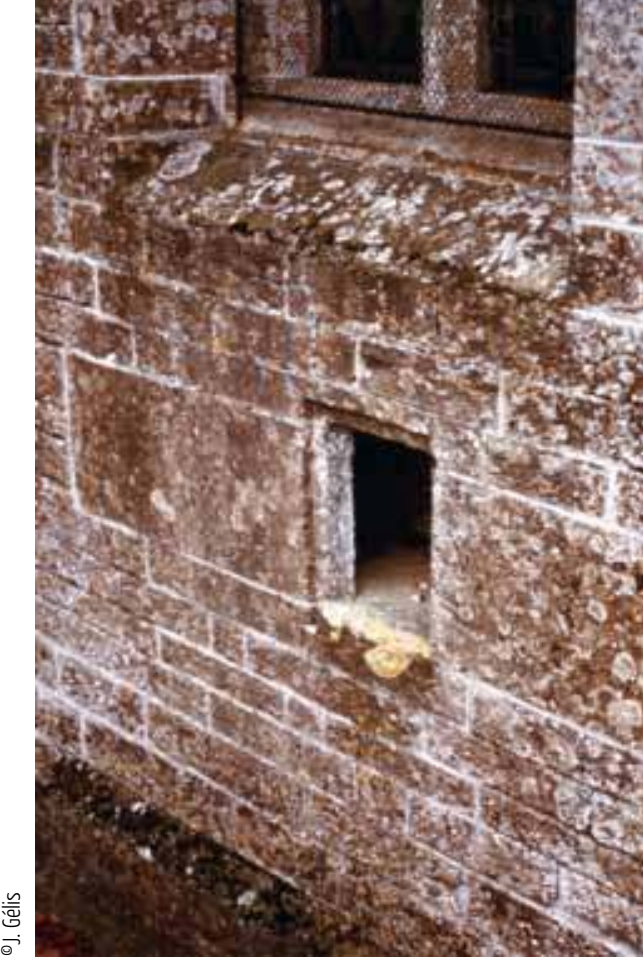

Une pratique dérobée

À l'extérieur du sanctuaire, juste derrière la statue miraculeuse, avait été aménagée « la loge des enfants morts sans baptême ». C'est là qu'était déposé le cadavre de l'innocent après que l'Église ait interdit de l'exposer à l'intérieur du lieu saint. Chapelle du cimetière de Pouilly-en-Auxois (Côte-d'Or). du lieu saint, les pèlerins, se dirigent vers la chapelle où est installée la statue de la Vierge miraculeuse. Ils déposent le corps sur l'autel ou sur le marche-pied de l'autel, parfois sur une table installée à proximité.

Le petit corps est alors démailloté et c'est donc dénudé et couché sur le dos qu'il est exposé devant l'image. La rigidité du cadavre empêche parfois de procéder à une présentation correcte du corps: impossible d'allonger les jambes et de joindre les mains de l'enfant... Autour de sa dépouille, les pèlerins, accompagnés maintenant de personnes pieuses du lieu assemblées à son de cloche, commencent à implorer la Vierge. Tous sont conscients de la gravité du moment. L'attente commence... On prie, on allume des cierges, on fait dire des messes, on chante les litanies de la Vierge tout en surveillant attentivement le petit cadavre. Mieux vaudrait d'ailleurs dire les petits cadavres lorsqu'il s'agit d'un sanctuaire très fréquenté: ainsi, à Moustiers-Sainte-Marie, voit-on couramment deux, trois, voire quatre enfants alignés autour desquels s'affairent les pèlerins (Gélis 1993). Tous détaillent des yeux ces corps dont on espère qu'ils vont revenir à la vie... Lequel sera le premier à bénéficier d'une grâce ? Il arrive souvent que le curé intervienne pour faire retirer l'un des cadavres qui entre décidément en putréfaction et incommode l'assistance; les personnes qui ont amené les autres enfants s'en trouvent confortées dans leur espoir de réussir puisque les autres petits corps sont intacts... Et voici que l'un des fidèles, plus attentif sans doute que les autres, commence à observer le début d'un changement sur le cadavre d'un innocent. Il s'empresse d'annoncer à tous la nouvelle, car il y a une grande fierté à être le premier à pressentir le retour de la vie dans un corps inerte. La tension en effet n'a cessé de croître dans ce huis clos paradoxal réunissant autour du cadavre d'un enfant des hommes et des femmes venus d'horizons divers et que le hasard a assemblés là. 


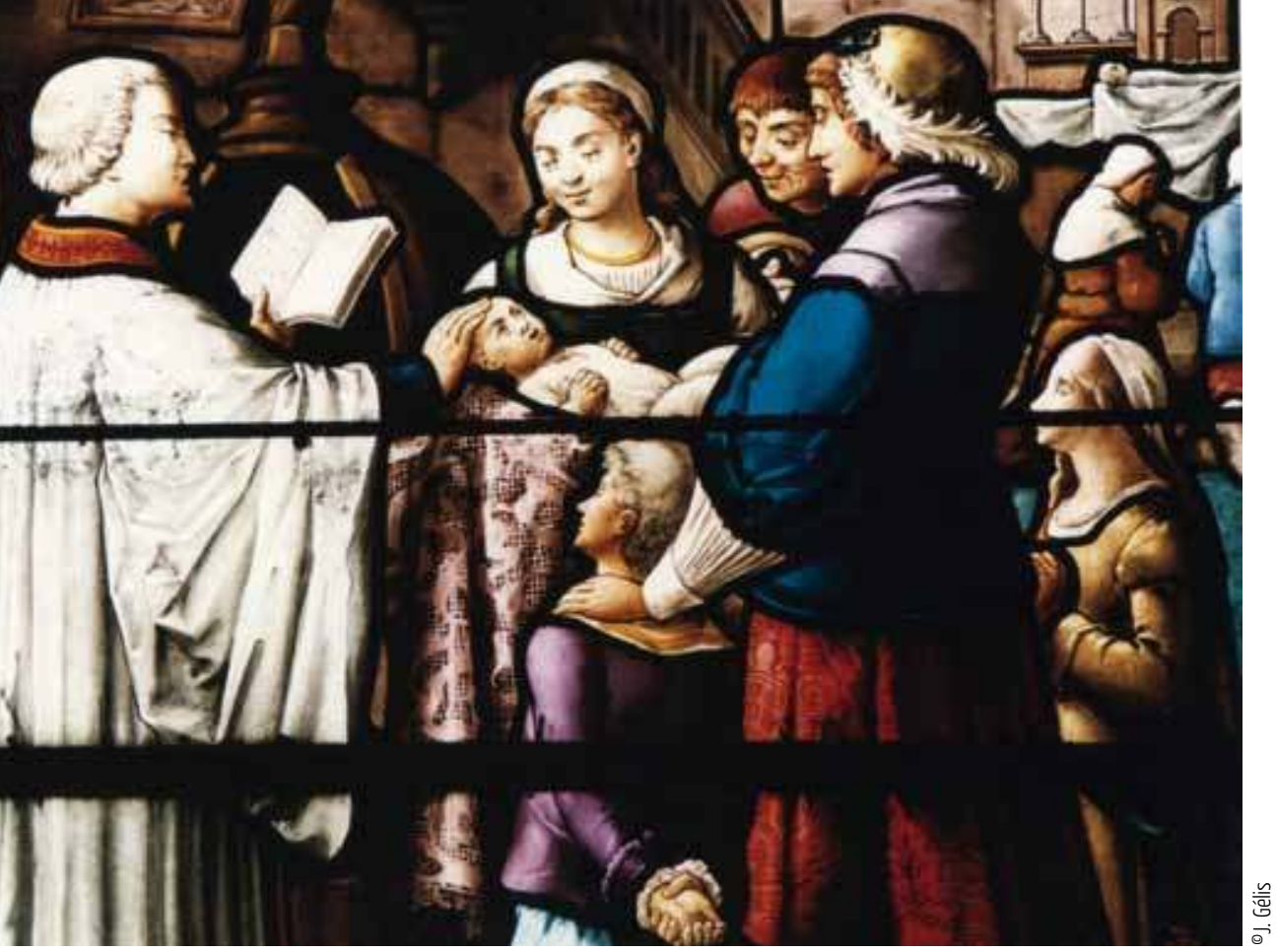

Deux temps du miracle

Au fond du sanctuaire, des pèlerins recueillis prient au pied de l'image miraculeuse, alors que le mort-né est exposé sur l'autel. Après le miracle, le prêtre procède au baptême de l'enfant. Alors que les pratiques de répit ont quasiment disparu, l'Église en cette fin du xixe siècle, célèbre un miracle vieux de deux siècles qui contribue maintenant à l'exaltation du culte marial. Notre-Dame des Fleurs, Villembray (Oise)

Vitrail de l'atelier Roussel, Beauvais, 1888 (détail). curé qui encourageait l'exposition du corps des enfants mort-nés dans son sanctuaire remarque, en arrivant à l'église, que « tout le corps changeoit en coulleur vermeille». Lui posant la main sur le ventre, il lui trouve une chaleur modérée et quelques minutes plus tard « chaud et en sueur ». L'enfant à qui on avait croisé les mains sur l'estomac les détache : elles glissent le long du corps et tombent sur la table. Et voilà que la plume que l'on avait posée sur ses lèvres se met à bouger, « haussant et se baissant plusieurs fois (...), de quoi il fut jugé que l'enfant soupiroit ». C'est alors que la créature remet ses mains sur son estomac, avant de les laisser pendre à nouveau le long de son corps. Le curé qui relate les faits rapporte ensuite qu'il « vit l'enfant pousser trois soupirs et la poitrine s'eslever et la veine qui traverse le front d'une coulleur rouge et battante». Ne doutant plus décidément de la réalité des signes de vie, le curé «baptisat sous condition ledit enfant $»$ (Montulet-Henneau 1986). Alors que les pratiques de répit ont quasiment disparu, l'Église en cette fin du xix siècle, célèbre un miracle vieux de deux siècles qui contribue maintenant à l'exaltation du culte marial.

Il ne s'agit pas de se prononcer sur la réalité du miracle ou de s'interroger sur une éventuelle manipulation, mais de prendre en considération une pratique populaire aujourd'hui oubliée. Des milliers et des milliers d'embryons et d'enfants à terme ont été ainsi exposés et ont donné des « signes de vie » entre le xiv et le xix ${ }^{e}$ siècle dans des dizaines de sanctuaires en France, en Belgique, en Suisse, en Autriche, en Allemagne du sud et en Italie du nord.

Examinons de plus près ce rituel du désespoir. Exposer un corps mort devant une image miraculeuse n'est pas un geste exceptionnel en ces temps de foi. Les annales des sanctuaires relatent des cas de retour durable à la vie de personnes que l'on avait cru mortes des suites d'un accident. Et les textes hagiographiques reprennent constamment, en citant les miracles du Christ et des saints, des cas de retours définitifs à la vie, les plus emblématiques étant sans doute ceux du Lazare sortant de son tombeau et de la fille de Jaïre se redressant sur la couche où on l'avait vue morte. Et puisque ces retours 
en arrière, de la mort à la vie, sont jugés possibles, à l'image du modèle évangélique, pourquoi le retour temporaire à la vie d'un enfant mort-né ne le serait-il pas ? Pourquoi ne pas espérer l'impossible ici et maintenant?

\section{Des « signes de vie » ? Quels « signes de vie » ?}

Les « signes de vie » doivent être abordés dans une double perspective. Ils s'inscrivent d'abord dans une géographie du corps de l'enfant. Mais si toutes les parties sont affectées, elles réagissent différemment selon l'âge du foetus à la naissance: enfant à terme ou avorton de quelques mois de conception. L'apparition de ces signes obéit également à des facteurs externes: température ambiante et temps écoulé depuis l'accouchement. La froidure de l'hiver peut en effet favoriser la conservation des corps.

La morphologie des signes de vie est aujourd'hui bien connue. Pour l'assistance, il ne fait aucun doute que le principe vital réside dans la tête et la poitrine; ce sont donc les parties du corps que l'on surveille et que l'on touche fréquemment. C'est habituellement le changement de couleur à la face, au ventre ou à la poitrine qui annonce la mutation de l'état du corps. Il s'agit toujours d'une couleur vive qui tranche avec la teinte cadavéreuse que l'enfant présentait à son arrivée au sanctuaire: « De pâle bleu que ses lèvres étaient, lui sont devenues entièrement vermeilles et rouges comme du sang ». Ce premier signe s'accompagne fréquemment de chaleur. Puis d'autres manifestations apparaissent. Les assistants « sentent le corps et principalement la tête dudit enfant être chaude et la veine de la tempe battre ». Ils se persuadent alors que le vie interne reprend son cours puisque le pouls se rétablit. Et voilà que la respiration semble réapparaître: on sent « les souffles sensibles de son haleine »... Des épanchements aqueux accompagnent ces manifestations: de la salive, des larmes, une sueur abondante. Du sang coule par les narines, l'oreille ou le nombril; or, plus que tout autre signe, le sang est aux yeux des assistants le symbole de la vie. Et puis il y a les mouvements qui agitent le corps; les bras et les jambes qui changent de place, et surtout l'ouverture des paupières... Ces hommes et ces femmes harassés de fatigue, après des jours et des jours passés à veiller le corps ont beau se rendre compte qu'ils ne parviennent pas à croiser le regard vide de l'enfant, ils ne veulent retenir de cet instant que le sentiment exquis d'avoir enfin atteint le but: leur foi persévérante a triomphé de l'adversité... De cet enfant sans âme, on a fait un « enfant du Ciel » puisqu'il est sauvé! Comment devant ces « preuves » éclatantes, plus «évidentes » les unes que les autres, les assistants pourraientils douter un instant de la « réalité » du miracle ? Le caractère spectaculaire de ce « retour en arrière », contre toute logique humaine qu'est le "répit», la fréquente dramatisation de la scène, tout contribue à faire du miracle des mort-nés un miracle sans pareil! La manifestation des « signes de vie » peut être 
accrédité l'idée que, décidément, quelque chose d'extraordinaire se produisait. C'est dans ce contexte d'attente angoissée et d'exaspération des sentiments que s'inscrit l'appel au corps médical.

Matrones, chirurgiens ou médecins, sont invité à témoigner de la réalité des « signes de vie » que les assistants voient apparaître sur le corps de l'enfant. Le comportement de la matrone est loin d'être uniforme. Le plus souvent pourtant, on la voit jouer un rôle actif au sein du groupe de femmes qui implorent la Vierge ou le saint intercesseur, mais dans certains sanctuaires, elle reste au contraire plus extérieure aux évènements. Elle surveille, juge et confère « le petit baptême » si on le lui demande. Le témoignage de l'homme de l'art ou de l'accoucheuse est, à l'évidence, attendu des contemporains, car l'avis que ceux-ci donnent est capital pour assurer la validation du cas et éviter un litige ultérieur. Leur fonction, leur expérience des accouchements les amènent tout naturellement à s'exprimer, en tant que spécialistes du corps, sur les signes de vie et de mort constatés successivement chez un nouveau-né. À vrai dire, il s'agit d'une démarche inhabituelle puisque ce que l'on attend tout de même des praticiens c'est qu'ils sachent reconnaître des signes de mort après la vie, et non pas l'inverse.

En général, le praticien n'assiste pas à la première phase du « répit »; il ne délivre pas de certificat de décès; on ne fait appel à lui que pour les phases deux et trois, c'est à dire pour établir le constat des « signes de vie » et de la « deuxième mort », définitive celle-là. Il existe pourtant une exception à cette règle: dans les cas d'infanticide. Le chirurgien ou la sage-femme procèdent alors à l'examen du corps mort, et en communiquent le résultat oralement (pour la sage-femme) ou par écrit (pour le praticien) aux autorités religieuse et

L'enfant donne des « signes de vie » Allongé sur un coussin et paré de fleurs, l'enfant est figuré devant l'image, au moment précis du « répit », alors qu'il vient d'ouvrir un œil... Cette mise en scène ressemble beaucoup aux ostensions d'enfants suisses et italiens morts en très bas âge dans la première moitié du xxe siècle. Chapelle Notre-Dame des Sept Douleurs. Ex-voto, 1750 (détail).

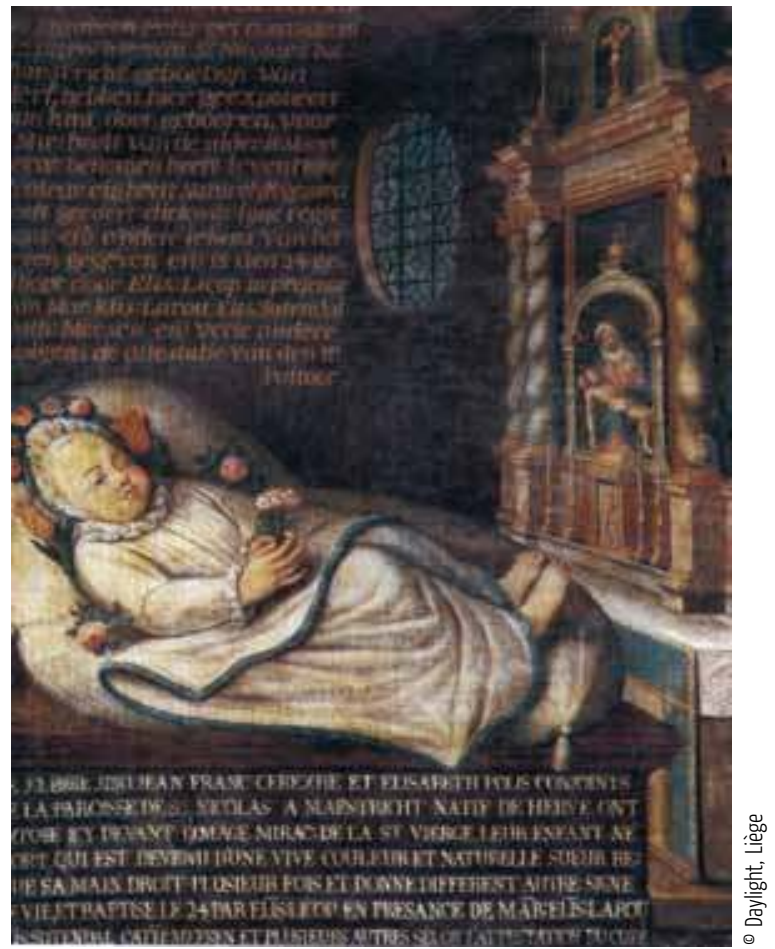

Un Cadavre qui donne des « signes de vie» 
du modèle mécaniste, qui respectait « l'incontestable » dogme religieux, fait place à la mort-processus de l'école vitaliste, dont l'énoncé doctrinal trouve sa perfection avec Bichat: la mort est un processus complexe, multiple, qui s'étend dans le temps, et qui est donc morcelé (Milanesi 1991).

Cette évolution de l'énoncé sert de toile de fond aux débats sur le cas des mortnés. Paradoxalement, en introduisant davantage de souplesse dans l'explication de la mort, la théorie vitaliste, et avec elle le corps médical, accrédite la thèse du « répit » possible: le jeune corps paraît mort et ne l'est peut-être pas. La mort-processus est réversible et la porte reste donc ouverte à un retour à la vie... Le petit miraculé entre dans la catégorie des individus qui ont eu une mort imparfaite.

Le cadavre du mort-né est un cadavre sans statut et c'est bien cette absence de statut qui inquiète les parents car, par-delà la question du baptême, elle peut entraîner des contestations au sein des familles. Qu'il ait manifesté quelque signe de vie et voilà qu'il peut être considéré comme un héritier, même passager. Pour le père dont la femme est morte en couches, ou la mère qui a perdu son mari pendant sa grossesse, c'est son propre avenir qui peut être affecté selon que l'enfant a ou non manifesté sa vitalité. Derrière le problème du baptême, on devine donc des questions qui relèvent du droit privé.

\section{La sépulture de l'enfant miraculé}

Mauvaise naissance, corps mis à l'écart, âme errante: telle était la vision de la destinée de l'enfant mort-né. À l'inverse, l'enfant ondoyé après un répit, puis « retourné à mort » était censé intégrer sa communauté. Or dans la pratique, le corps n'était généralement pas ramené à son lieu d'origine; il paraissait normal de le laisser au lieu même où la grâce s'était manifestée et ce choix était ressenti comme un bienfait supplémentaire. La sépulture ad sanctos du petit cadavre était pour la parenté la plus belle des récompenses. Lorsque le " sanctuaire à répit » était une église paroissiale, les petites dépouilles reposaient à l'écart dans le cimetière: un « rang spécial » leur était réservé. Lorsqu'il s'agissait d’une chapelle de confins de terroirs, un « cimetière de bébés » avait été sommairement aménagé : ainsi, à Notre-Dame du Chemin à Serrigny en Bourgogne, à Nanc, près de Saint-Amour dans le Jura ou à Notre-Dame de l'Arbrisseau à Salles près de Chimay dans le Hainaut belge. Parfois, les petits corps étaient ensevelis dans un caveau de la chapelle, comme à Viserny près de Montbard, en Côte d'Or (Gélis 2006). Quelques-uns subsistent aujourd'hui alors même que leur mémoire s'est perdue.

\section{L'enseignement des fouilles}

Rares sont les comptes rendus de fouilles effectuées dans le passé qui précisent ce que furent les conditions de sépulture. La plupart des sites ont souvent été prospectés assez superficiellement. Seul le hasard pouvait laisser espérer la sauvegarde de l'un d'eux. Ce fut le cas en 1992 à Oberbüren, dans le canton de Berne en Suisse. Situé sur une colline dominant une confluence, au point de contact entre les trois anciens diocèses de Berne, Lausanne et Constance, cet ancien lieu de pèlerinage était devenu un enjeu entre catholiques et protestants au moment de la Réforme. Les cinq campagnes de fouilles qui se sont succédées de 1993 à 1997 ont permis d'éclairer sous un jour nouveau les conditions 


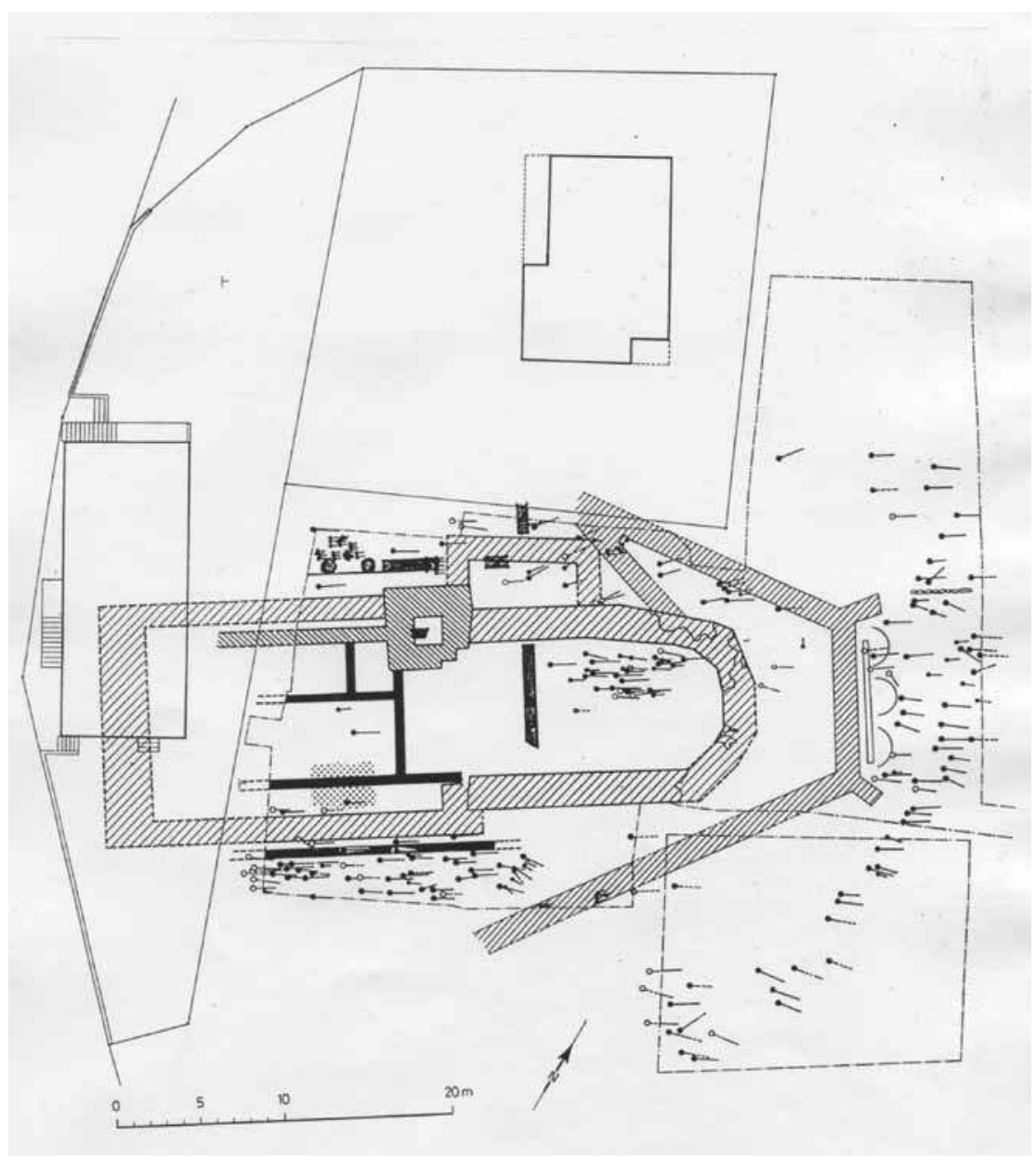

๑ Plan par H. Kellenberg, Service archéologique du canton de Berne.

\section{Plan du site d'Oberbüren}

Sur un promontoire situé à

une confluence se dressait la chapelle

de pèlerinage d'Oberbüren, haut lieu

des pratiques de « répit »

dans la seconde moitié du xve siècle.

Ce plan levé au cours des fouilles

en 1994 montre la répartition

des corps. Ceux des enfants

miraculés, fœetus abortifs

ou enfants à terme, sont proches

des flancs du sanctuaire,

disposés comme dans un charnier,

serrés les uns contre les autres

en plusieurs lits superposés.

d'ensevelissement des enfants mortnés miraculés dans ce sanctuaire qui eut un grand rayonnement de la fin $\mathrm{du} \mathrm{Xv}^{\mathrm{e}}$ siècle aux années 1530, date à laquelle les protestants rasèrent le sanctuaire (Ulrich-Boschler \& al. 2008).

La fouille du site a permis d'éclairer les conditions d'ensevelissement des mort-nés miraculés. On a retrouvé 490 tombes et les restes de 550 individus, dont 250 enfants (alors qu'au moins 2000 enfants semblent avoir été miraculés pendant la période considérée). Les squelettes d'enfants gisaient pour partie dans des sépultures individuelles, pour partie, la plus nombreuse, dans des tombes collectives. Aucun matériel n’a été retrouvé et tous semblent avoir été ensevelis dans leurs langes ou dans un linceul. Comme la place était comptée, en raison de l'étroitesse du site, on avait pris l'habitude de superposer les corps sur plusieurs niveaux. On procédait de la manière suivante: on creusait d'abord une grande fosse, puis on disposait les enfants corps contre corps, au fur et à mesure qu'on les amenait; enfin, on les recouvrait de terre. Quand un premier lit était constitué, on en disposait un autre par-dessus et lorsque le trou était plein, on le rebouchait et on creusait un peu plus loin. On pouvait ainsi avoir quatre lits de corps superposés. Tous les innocents étaient enterrés la tête à l'ouest. Un certain nombre d'entre eux reposait sur le dos et avait les bras repliés sur l'abdomen, mais beaucoup étaient couchés sur le côté, les jambes repliées, en position embryonnaire. D'autres avaient le corps contorsionné et les épaules paraissaient avoir été comprimées. Il est possible qu'un fossoyeur ait été affecté au sanctuaire et comme il disposait de peu de place, il serrait les corps les uns contre les autres sans trop se préoccuper de leur position.

Tous ces enfants étaient des bébés morts avant, pendant ou juste après leur naissance. Les anthropologues qui ont procédé à l'étude scientifique des ossements ont retenu la taille des squelettes comme critère de détermination de l'âge des enfants. Ils ont considéré qu'entre 45 et 55 centimètres ils avaient à faire à des enfants à terme et qu'au-dessous 
de 45 centimètres il s'agissait de fœtus expulsés prématurément. Même si ces critères sont discutables (pourquoi ne pas avoir pris comme référence la qualité des synostoses, ces soudures des extrémités des os dont on sait qu'elles interviennent à des stades différents de la croissance selon les os concernés), ils apportent de précieux renseignements sur les enfants miraculés. Plus du tiers d'entre eux étaient des prématurés et les plus petits fotus étaient des avortons de quatre à cinq mois de conception. Mais il est vrai que les interruptions involontaires de grossesse étaient nombreuses aux siècles classiques: les accidents, le surmenage des femmes pendant la grossesse, les carences et les insuffisances alimentaires aboutissaient fréquemment chez la femme enceinte à l'expulsion prématurée de l'embryon.

Tous ces enfants avaient au moins la chance d'être sauvés et de mériter une sépulture en terre consacrée, à la grande satisfaction des parents qui remerciaient en offrant un ex-voto

Mais qu'en était-il des autres, de tous ceux qui ne donnaient pas de « signes de vie » et dont il est d'ailleurs bien difficile d'évaluer le nombre ? Nous savons qu'à l'abbaye d'Ursberg en Souabe, qui fut sans doute le plus grand sanctuaire à répit d'Europe puisqu'on y amenait annuellement à la fin du xviie siècle jusqu'à 2000 enfants mortnés, près de la moitié ne donna jamais de « signes de vie » (Gélis 1998). En cas d'échec, les parents se mettaient alors en quête d'un autre lieu d'exposition... Jusqu'au moment où la décomposition du corps les obligeait à l'enterrer clandestinement tout contre le mur d'un lieu de culte. Cette sépulture « sous l'égout du toit » fut fréquente en Alsace jusque dans les années 1880. Elle constituait un moyen empirique de sauver l'enfant, car on se persuadait que l'eau qui tombait sur le sanctuaire, alors qu'on procédait au baptême d'un enfant bien vivant, bénéficiait au pauvre innocent... On avait fait ce que l'on avait pu et Dieu pourvoirait bien au reste...

\section{Les hésitations de l'Église}

Un événement aussi spectaculaire et massif plaçait l'Église dans une situation inconfortable, car elle la divisait. Pendant des siècles, les clercs ont justifié ce miracle. Face aux protestants qui n'y voyaient que tromperie, ils en ont même fait une manifestation de la vraie foi. Ces pratiques n'ont d'ailleurs été condamnées par la 


\section{Remercier Dieu pour l'enfant qui survit}

Sur neuf enfants qui leur sont nés, les parents en conservent un...

Mais puisque l'avenir de la famille

paraît assuré, ils ne songent plus qu'à remercier Dieu: « Mon Dieu, huit enfants sont près de toi.

Je te remercie de m’avoir conservé

le neuvième.» Tableau votif,

Haute Autriche, Österreichisches Museum für Volkskunde, Vienne, 1775.

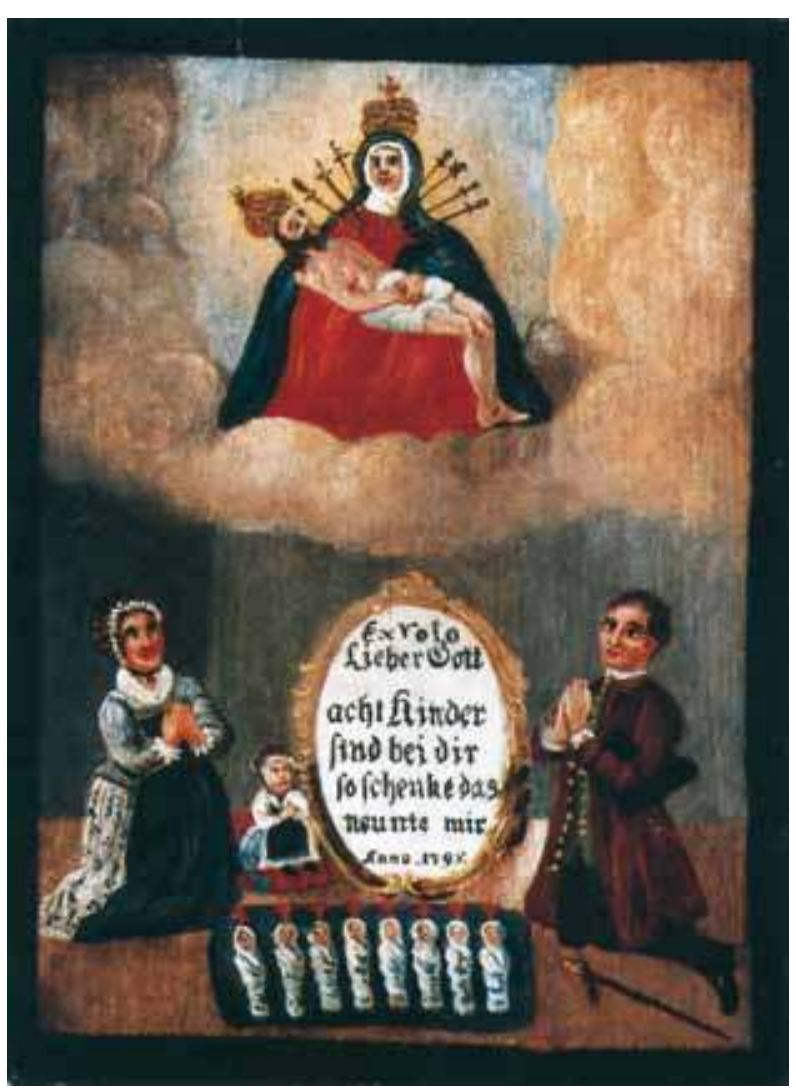

curie romaine qu'en 1729. Mais entre haut et bas clergé, entre réguliers qui y étaient favorables et évêques qui les condamnaient, les rapports ont été parfois tendus. Et de ce fait, bien des rituels ont été cachés à la hiérarchie.

Dans les années 1670-1680, on assiste un peu partout à une véritable épidémie de « répits » et la hiérarchie se rend compte de l'ambiguité de ce qui se passe. Sa condamnation du rite entraîne des conflits parfois sévères et des sanctions, mais la demande des populations est telle que le rituel persiste. Au xviII ${ }^{e}$ siècle, l'institution croit trouver une solution avec la césarienne sur femme morte préconisée par le Traité d'embryologie sacrée du jésuite italien Cangiamila. On attend la mort de la femme qui ne parvient pas à accoucher et on s'empresse d'inciser son cadavre pour tirer l'enfant vivant et l'ondoyer... Cette pratique que l'on veut systématique mais qui ne tient pas compte de la vie de la mère, va soulever l'opinion dans la seconde moitié du siècle et l'église tente alors d'y substituer le baptême intra-utérin.

$\mathrm{Au} \mathrm{XIV}{ }^{\mathrm{e}}$ siècle, la valorisation du culte marial conduit à l'acceptation désormais du « répit » par l'institution; ce miracle devient même le symbole de la toute puissance de Dieu. Ce n'est que dans les années 1950 que l'institution acceptera d'atténuer la peine imaginée pour les enfants morts sans baptême. Enfin, il y a quelques années une commission pontificale s'est penchée sur le concept de limbes pour souligner que ce ne fut jamais un dogme d'église, mais une position de circonstance. 


\section{Un cadavre de l'entre-deux}

Tous ces rites dérobés, toutes ces tentatives pour réintégrer un corps mort dans le cycle de vie communautaire renvoient en fait à des croyances sans doute antérieures à la christianisation des populations rurales. Dans la croyance populaire, on a pitié de l'enfant mort-né, ce qui ne l'empêche pas de le craindre. On se méfie de cet « esprit égaré », de cette « âme en peine » en perpétuel mouvement. On pense que le mort-né disparu « avant d'avoir fait son temps » est toujours prêt à reprocher à ses parents la triste condition qui est la sienne ? Il appartient en effet à la grande cohorte des réprouvés, de tous ceux qui ont eu une mauvaise mort et qui ne cessent d'importuner les vivants: la « chasse sauvage ». Dès lors on comprend mieux l'importance du rite destiné à réinsérer l'enfant non seulement dans la famille mais également dans la communauté, car ce cadavre embarrassant passe pour annoncer des calamités à venir, mauvaises récoltes et maladies du bétail.

Malgré leur habillage chrétien, les rites d'exposition et d'ensevelissement des enfants mort-nés laissent entrevoir une strate profonde de croyances fondée sur l'alliance étroite entre l'homme et la nature qui l'imprègne. Ils révèlent une manière d'être au monde et de mourir qui a progressivement disparu au cours du xixe siècle. Des dépôts retrouvés à proximité de certains « sanctuaires à répit » témoignent de l'ancienneté des pratiques sans que l'on puisse affirmer qu'il a existé une continuité sans faille du rituel d'exposition. C'étaient surtout les vieilles « pierres saintes » situées sur les limites de terroirs qui attiraient les pèlerins en quête de salut pour les petits enfants. On fréquentait volontiers ces «sanctuaires de la nature », ces « barques de pierre », ces « berceaux sarrasins», c'est-à-dire païens, auprès desquels on venait encore, il y a un siècle et demi, ensevelir clandestinement le petit cadavre. Il est probable qu'il s'agissait de cette antique « terre des morts », de ces lieux de sépulture primitifs que « la naissance du cimetière » (Lauwers 2005) imposé par l'Église à partir du x ${ }^{e}$ siècle fit progressivement disparaître: mais en avait-on jamais vraiment perdu le chemin?

Photo d'ouverture: L'Église ne craint plus le « répit » des enfants mort-nés. Elle en accepte d'autant plus aisément la représentation que ce miracle contribue désormais à l'essor d'une « religion en sentiment ». " Miracle de l'enfant ressuscité par saint Maxime », tableau de Patritti, 1846, cathédrale de Riez (Alpes de Haute Provence). 


\section{POUR CITER CET ARTICLE}

Gelis, J. 2013 Un Cadavre qui donne des «signes de vie». Le cas de l'enfant mort-né au sanctuaire à répit, in Guy, H. Jeanjean, A. \& Richier, A., Le Cadavre en procès, TechniquesE Culture 60 : 44-59.

\section{RÉFÉRENCES}

Bernos, M. 1973 Miracles chez les Servites en Provence à l'époque moderne, Revue d'histoire de la spiritualité, 49: 243-256.

- 1970 Réflexions sur un miracle de l'Annonciade d'Aix-en-Provence. Contribution à l'étude des «sanctuaires à répit », Annales du Midi: 5-20.

Carol, A. 2004 Les Médecins et la mort, XIX ${ }^{e}-X^{e}$ siècles. Paris: Aubier (Collection historique).

Coste, M.- C. (Dir.) 2011 Le Corps des anges: Actes de la journée d'études des pratiques funéraires autour de l'enfant mort au Moyen Âge. Milan: Silvana Editoriale.

Gélis, J. 2006 Les Enfants des limbes. Mort-nés et parents dans l'Europe chrétienne. Paris: Audibert.

- 1998 Lebenszeichen-Todeszeichen: Die Wundertaufe totgeborener Kinder im Deutschland der Aufklärung. In J. Schlumbohm, B. Duden \& J. Gélis, Rituale des Geburt. Eine Kulturgeschichte. Munich: Beck Verlag. 269-288.

— 1993 Les sanctuaires «à répit» des Alpes françaises et du Val d'Aoste: espace, chronologie, comportements pèlerins ». Archivio Storico Ticinense anno xxx: seconde série, nº 114: 183-222.

Lauwers, M. 2005 Naissance du cimetière. Lieux sacrés et terre des morts dans l'Occident médiéval. Paris: Aubier (Collection historique).

Lett, D. 1997 La Mort de l'enfant sans baptême. In: L'Enfant des miracles. Enfance et société au Moyen Âge (XII ${ }^{e}$-XIII ${ }^{e}$ siècles), Paris: Aubier (Collection historique) : 205-218.

Maillard, C. 1651 Histoire de Notre-Dame de Hal. Bruxelles: Hubert Antoine-Velpire.

Milanesi, C., 1991 Mort apparente, mort imparfaite: médecins et mentalités au XVIII siècle. Paris: Payot.

Montulet-Henneau, M.E. 1986 Un Village hesbignon aux portes du paradis; résurrections d'enfants mortnés au sanctuaire "à répit » de Moha (1707-1773), Annales du cercle hutois de sciences et beaux-arts XL: 109-179.

Renzetti, E. 1993 Pellegrini nelle morte. Risurrezioni temporanee battesini nei santuari del Tirolo, Archivio StoricovTicinense anno xxx: seconde série, $\mathrm{n}^{\circ} 114$ : 223-246.

Seidel Menchi, S. 2000 Les Pèlerinages des enfants mort-nés. Des rituels correctifs pour un dogme impopulaire? In P. Boutry, P.A. Fabre \& D. Julia, Rendre ses voeux; les identités pélerines dans l'Europe moderne (XVI'-XVIII e siècles). Paris: Éditions de l'EHESS : 139-154.

Ulrich-Boschler \& S., Gutscher, D. 1998 Wiedererweckung von Totgeborenen. Ein Schweizer Wallfahrtszentrum im Blick von Archäologie und Anthropologie. In J. Schlumbohm, B. Duden \& J. Gélis, Rituale des Geburt. Eine Kulturgeschichte. Munich: Beck Verlag. 244-268.

Winslow, J-B.\& Bruhier d'Ablaincourt, J.-J., 1742-1745 Dissertation sur l'incertitude des signes de la mort et l'abus des enterrements précipités, 2 vol., Paris: Audibert.

\section{RÉSUMÉ}

Un Cadavre qui donne des "signes de vie ». Le cas de l'enfant mort-né au sanctuaire à répit. La venue au monde d'un enfant mort-né a toujours constitué pour le couple un profond traumatisme. Aux siècles passés où la destinée de l'âme était si importante dans le monde catholique, les parents recouraient volontiers à l'exposition du petit cadavre devant une Vierge miraculeuse dans l'espoir de lui voir donner les «signes de vie » qui autorisaient le baptême salvateur: l'âme était sauvée et le cadavre pouvait alors être enterré en terre consacrée et non pas mis au rebut. Ce « répit » entre deux morts, puisque le corps de l'enfant redevenait cadavre après le miracle, se déroulait selon un rituel immuable. Dans le « sanctuaire à répit », pouvaient voisiner pendant des jours, voire des semaines, 
les dépouilles dénudées de plusieurs enfants exposés, dont les pèlerins surveillaient le comportement. Le retour de la chaleur et de la coloration du corps, les mouvements des membres, les épanchements aqueux et sanguins étaient interprétés comme des signes véritables de retour à la vie. À l'angoisse de la famille et de la communauté après la venue d'un enfant mort succédait l'enthousiasme des pèlerins. Le corps était ordinairement enterré dans le cimetière qui jouxtait le sanctuaire. Plus tard, les parents ne manqueraient pas de rendre hommage à la Vierge en offrant un ex-voto célébrant le miracle. Du XIv ${ }^{e}$ au XIv ${ }^{e}$ siècles, des milliers et des milliers de cadavres d'enfants mort-nés ont été ainsi exposés en France, Belgique, Suisse, Autriche, Allemagne du sud et Italie du nord. Si les praticiens (accoucheuses, chirurgiens et médecins) ont longtemps accepté de cautionner le miracle, ils commencent à prendre leurs distances à partir des années 1730-1740. Désormais, ce n'est plus le cadavre de l'enfant qui justifie leur intervention ; ce qu'ils veulent c'est sauver l'enfant à sa naissance. L'Église de son côté a toujours été très divisée sur l'attitude à tenir à l'égard des enfants mort-nés voués au néant. Autant les curés faisaient volontiers preuve de tolérance voire de complicité à l'égard des « répits », autant la hiérarchie se montrait méfiante à l'égard de pratiques qu'elle jugeait entachées de magie et de tromperie. En fait, derrière la question du sacrement chrétien se devinent des comportements qui ont longtemps caractérisé le monde rural : croyance à la « chasse sauvage » et volonté de restaurer par un rituel approprié l'équilibre un instant rompu de l'ordre du monde, puisque la naissance d'un enfant mort est toujours interprétée par la communauté comme le signe annonciateur de quelque calamité naturelle.

\section{ABSTRACT}

A corpse showing signs of life. The case of the stillborn child and the respite sanctuary. The birth of a stillborn child has always been deeply traumatic for parents. In past centuries, when the Catholic Church regarded the destiny of the soul as highly important, parents would willingly expose the little corpse to the miraculous Virgin in the anticipation of signs of life which would allow for a saving baptism : its soul was saved et and the little corpse could then be buried in consecrated ground rather than being discarded. This 'respite' or period of limbo between two deaths was dealt with according to an immutable ritual.

The remains of several children would remain in a respite sanctuary during days or even weeks, while pilgrims surveyed their behaviour. Any changes to the appearance of the body such as the release of bodily fluids or the perception of heat and colour were considered as signs of a return of life. The anguish of the family or the community after the birth of the stillborn child would then be replaced by the renewed enthusiasm of the religious community. The body was normally buried in the graveyard next to the sanctuary. Later on, the parents would ensure to pay tribute to the Virgin Mary by offering an ex-voto in celebraion of the miracle. From 14th to 19th century, many thousands of children corpses have been exposed in France, Belgium, Switzerland, Austria, southern Germany and northern Italy. Although medical practitioners (midwives, surgeons, doctors) remained sympathetic to the miracle for a long time, their position changed from around 1730-1740 onwards. Henceforth, saving the child at birth became the priority rather than simply post-mortem medical intervention.

The Catholic Church, on the other hand, have always been ambiguous on what stance to adopt regarding the fate of the condemned souls of stillborn children. While parish priests could often show tolerance and even complicity towards 'respite' pratices, the ecclesiastical hierarchy were mistrustful and judged them as being tainted by occultism and delusion. Besides the importance of the Christian sacraments, these long lasting behaviours of rural society.

\section{MOTS CLÉS}

mort-né, baptême, miracle, signes de vie, signes de mort, sépulture

\section{KEYWORDS}

stillborn, baptism, miracle, signs of life, signs of death, grave 